Relations industrielles

Industrial Relations

\title{
Les tenants et les aboutissants de la convention collective
}

\section{Fernand Morin}

Volume 40, numéro 2, 1985

URI : https://id.erudit.org/iderudit/050140ar

DOI : https://doi.org/10.7202/050140ar

Aller au sommaire du numéro

Éditeur(s)

Département des relations industrielles de l'Université Laval

ISSN

0034-379X (imprimé)

1703-8138 (numérique)

Découvrir la revue

Citer cet article

Morin, F. (1985). Les tenants et les aboutissants de la convention collective. Relations industrielles / Industrial Relations, 40(2), 371-378.

https://doi.org/10.7202/050140ar
Résumé de l'article

Les tenants et aboutissants de la convention collective
Tous droits réservés (c) Département des relations industrielles de l'Université Laval, 1985
Ce document est protégé par la loi sur le droit d'auteur. L'utilisation des services d'Érudit (y compris la reproduction) est assujettie à sa politique d'utilisation que vous pouvez consulter en ligne.

https://apropos.erudit.org/fr/usagers/politique-dutilisation/ 


\title{
Les tenants et aboutissants de la convention collective
}

\author{
Fernand Morin
}

La durée de la convention collective constitue un point d'ancrage important des rapports collectifs du travail selon l'aménagement imposé au Code du travail (Québec). Ne faut-il pas savoir si !es parties ont ou n'ont pas conclu une convention collective, si elle lie encore les parties, quelle est la date de son dépôt, quelle est sa durée et la date de son expiration pour pouvoir répondre à ces multiples questions:

- un autre syndicat peut-il régulièrement être accrédité (art. 22 C.t.)?

- l'employeur peut-il demander la vérification de la représentativité du syndicat accrédité (art. 41 C.t.)?

- l'employeur peut-il unilatéralement modifier les conditions de travail (art. 59 C.t.)?

- l'une des deux parties peut-elle prendre l'initiative d'entamer la négociation (art. 52 C.t.)?

- l'une et l'autre parties peuvent-elles exercer des moyens de pression (grève et lock-out) (art. 107 C.t.)?

- la mésentente entre les parties constitue-t-elle un grief soumis au régime arbitral des art. 100 et s.s. C.t.?

Ces quelques exemples illustrent bien que le régime institué au Code du travail est axé sur l'existence ou non d'une convention collective. Cette situation ne peut surprendre, puisque la stabilité relative des rapports collectifs du travail constitue un des objectifs principaux du régime (la paix industrielle). N'est-ce pas aussi pour cette même raison que le législateur exige que la convention collective soit d'une durée déterminée ou déterminable d'au moins 1 an et d'au plus 3 ans (art. 65 et 66 C.t.), qu'en cas de doute quant à sa durée, le commissaire du travail peut la préciser (art. 52.2 C.t.) et que cette convention doit être publique et n'avoir d'effet qu'à compter de son dépôt ( $\operatorname{art.} 72$ C.t.)? Le législateur ajouta même une règle dérogatoire au régime général du droit, à savoir que la partie résiduaire d'une convention collective subsisterait, bien que «... une ou plusieurs de ces clauses» soient nulles ou annulées (art. 64 C.t.).

Le tribunal du travail eut récemment l'occasion de prendre position à l'égard de plusieurs des sous-questions rattachées à la durée de la convention collective ${ }^{1}$. Nous rappelons succinctement cette affaire et rapportons

* MORIN, Fernand, professeur, Département des relations industrielles, Université Laval.

1 Ambulance S.O.S. Enr. c. Rassemblement des employés techniciens ambulanciers du Québec et Union des camionneurs de construction et approvisionnement, mécaniciens d'autos, etc.; décision du 13 septembre 1984, no 500-28-000616-847. 
les éléments pertinents de la décision du tribunal que nous apprécions et commentons, par la suite.

\section{L'AFFAIRE AMBULANCE S.O.S.}

Un premier syndicat accrédité concluait, le 24 novembre 1981, une convention collective qui aurait été déposée (art. 72 C.t.) en décembre 1981. Les parties entendaient y être liées du ler février 1981 au ler avril 1984 (clause 20-01).

En novembre 1983, un deuxième syndicat soumettait une requête en accréditation qui fut reçue favorablement le 23 mars 1984 . L'employeur contesta auprès du tribunal du travail la valeur de cette nouvelle accréditation au motif que la requête était prématurée, c'est-à-dire présentée avant l'arrivée du délai arrêté à l'art. 22, par. d C.t.

\section{DÉCISION DU TRIBUNAL DU TRAVAIL}

M. le juge Bernard Lesage répondit à plusieurs sous-questions que soulèvent pareille situation et que nous rappelons d'abord à l'aide de quelques extraits:

Les parties peuvent-elles conférer un effet rétroactif à leur convention collective? Å cette question générale ou de principe, le tribunal répondit: «... l'économie générale des relations de travail a toujours voulu que les conventions collectives et sentences arbitrales puissent être rétroactives» (p. 8);

Ayant ainsi le pouvoir de régler postérieurement certaines situations, le tribunal du travail reconnut aussi que ces effets rétroactifs pouvaient bénéficier aux salariés d'alors même s'ils n'en n'avaient plus le titre au moment de la conclusion de la convention collective. Le principe de la rétroactivité étant reconnu, dit le tribunal, «... il n'est pas de raison de refuser à la fiction juridique toute sa portée. Ce pourrait aller, encore que le problème ne se soulève pas ici, à reconnaître des avantages à des salariés ayant perdu tel statut lors de la signature» (p. 10);

Selon l'art. 59 C.t. et pour les fins qui y sont visées, la convention collective survit-elle à son arrivée à terme ou est-ce seulement certains de ses effets qui y sont maintenus? Le tribunal du travail donna cette réponse: «Il n'y a aucune discussion possible alors sur la question de savoir si la convention collective perdure formellement après cette étape. La réponse est nécessairement négative puisque le deuxième paragraphe de cet article 59 se lit comme suit: "Il en est de même à compter de l'expiration de la convention collective et tant que le droit au lock-out n'est pas acquis ou qu'une sentence arbitrale n'est pas intervenue. Ce sont donc les conditions de travail apparaissant à la convention collective, par opposition au cortex de celle-ci, qui subsiste pendant un temps». (p. 6);

Lorsqu'il y est établi certains effets rétroactifs à la convention collective, la durée formelle de cette dernière commence-t-elle à compter de la date première de ces effets rétroactifs? Pour répondre à cette question, le tribunal 
du travail signala d'abord le nouveau libellé du deuxième paragraphe de l'art. 72: «Ce dépôt a un effet rétroactif à la date prévue dans la convention collective pour son entrée en vigueur ou, à défaut, à la date de la signature de la convention collective». Par cette dernière disposition, toujours selon le tribunal, «... Le législateur a ainsi clarifié dans une large mesure les modalités de cette pratique généralisée et de faire rétroagir une convention collective. À mon sens, ce paragraphe signifie qu'il est fort possible aux parties non seulement de régler des situations passées et de donner des effets rétroactifs à certaines conditions de travail, mais bien de faire rétroagir formellement toute la convention collective qui débute alors, par une fiction de la loi, à une date antérieure à sa survenance dans le temps réel. Tout dépendra de la phraséologie utilisée quant au traitement de la rétroactivité, et les négociateurs de cette clause en ont toujours été conscients bien naturellement.» (p. 9) De plus, le juge Lesage proposait de distinguer deux (2) situations. Si seulement certaines conditions de travail doivent produire un effet rétroactif, alors la durée même de la convention collective n'en serait pas affectée, mais «... Lorsque c'est tout le contenu de la convention qui est conventionnellement décrété s'appliquer à une date précise, il faut bien conclure en droit que c'est la durée de cette convention qui commence à cette date certaine et déterminée». (p. 7)

Une convention collective d'une durée excédentaire à trois ans doit-elle être considérée comme si elle n'avait pas été déposée? Notons qu'une réponse positive à cette question permettrait à un autre syndicat de soumettre valablement une requête en accréditation en pareille situation (art. 72, al. 3 C.t.). Le tribunal refusa cette approche catégorique: «Sans avoir à s'immiscer dans le domaine contractuel réservé aux parties, il est tout à fait possible de donner effet à la volonté du législateur en réduisant à la limite maximale possible les conséquences légales qu'on a cherché à faire déborder.» (p. 14) En l'espèce, le tribunal considéra que le début formel de cette convention collective devait être le ler février 1981: «... En venant à la conclusion ici que la convention excède d'un mois et demi la limite maximale de trois ans, devons-nous conclure qu'elle est entièrement nulle ou qu'elle n'est valide et en vigueur comme si son terme était ramené aux trois premières années?» (p. 11). Le tribunal retint ce dernier volet et déclara que cette convention collective ne pouvait dépasser le 31 janvier 1984 et qu'en conséquence, la requête en accréditation du 29 novembre 1983 était recevable selon l'art. 22 , par. d du Code du travail.

Profitant de l'occasion puisque l'affaire sous étude ne soulevait nullement ce point, le juge Lesage fit connaître son opinion «... pour les situations futures» au sujet d'une convention collective inférieure à la durée minimale d'un an (art. 65 C.t.). Selon le tribunal, une telle situation serait fatale et irrémédiable: «Il s'ensuit que lorsque le premier volet de l'exigence de l'art. 65 C.t. n'est pas rencontré, à savoir une convention collective d'au moins 1 an, la conséquence en est la nullité totale. Non seulement il ne serait être question pour un organisme quasi-judiciaire de se substituer à la volonté des parties pour édicter des conditions de travail alors qu'il y a des règles strictes pour l'intervention d'un arbitre de différend, mais il doit être constaté que l'accréditation n'a pas été utilisée minimalement. Il ne saurait être question d'invoquer l'art. 64 C.t. qui stipule qu'une convention collective n'est pas invalidée par la nullité d'une ou de plusieurs de ses clauses.» (p. 13). On 
ajoute aussi que le dépôt d'un tel acte ne modifierait en rien la situation puisque $« \ldots$ La doctrine et la jurisprudence sont stables à l'effet que le dépôt n'est qu'une formalité administrative, qui ne peut même être refusé lorsque certaines conditions de forme sont remplies et qui ne fait certes pas foi de la validité du document, lorsqu'il y a lieu». (p. 13)

\section{COMMENTAIRES}

Comme le rappella, à quelques reprises, le juge Lesage, la liberté des parties de donner un effet rétroactif aux dispositions de leur convention collective fut depuis longtemps reconnue par les tribunaux. Quoiqu'il en soit, il s'agit d'une nécessité pratique inéluctable. Le deuxième paragraphe de l'art. 72 précise même que les effets de la convention collective seraient nécessairement rétroactifs à la date de sa conclusion formelle du seul fait de son dépôt. On pourrait même soutenir que les parties ne pourraient convenir que leur convention collective puisse prendre strictement effet au seul jour de son dépôt. Ce jugement traite également de deux questions particulières que nous voulons commenter: la computation possible du temps rétroactif pour établir la durée totale de la convention collective et la valeur juridique d'une convention collective conclue pour une durée inférieure à un (1) an.

\section{Le temps rétroactif est-il de la durée de la convention collective?}

M. le juge Lesage répondit positivement à cette question dans le cas où les parties entendent donner un effet rétroactif à toute la convention collective. Le tribunal reconnut cependant qu'en pratique bien des dispositions ne pouvaient produire pareil effet ${ }^{2}$. En tout respect pour cette façon de voir, nous croyons que l'économie générale du régime des rapports collectifs établi au Code demande que seul le temps «futur» d'une convention collective soit retenu pour établir sa durée réelle. En clair, la durée d'une convention collective débuterait le jour de son dépôt. À l'appui de cette assertion nous soumettons cette argumentation en sept (7) points qui se complètent les uns les autres:

1. Selon l'approche maintenant retenue par le tribunal du travail, il suffirait aux parties de déclarer quelques dispositions de la convention collective non rétroactives et même celles qui ne peuvent l'être de toute manière pour éviter le calcul rétroactif de sa durée totale et gagner ainsi du temps? Notons que l'inverse serait aussi exact. Un libellé malencontreux au sujet de la rétroactivité pourrait réduire d'autant l'acte entier. N'est-ce pas tomber dans un formalisme dangereux et contraire à la sécurité juridique pourtant nécessaire aux parties à la négociation collective?

2. La durée maximale de trois (3) ans (art. 65 C.t.) n'a rien de magique et répond principalement de l'expérience pratique. Elle constitue un délai suffisant pour que les parties bénéficient d'une stabilité acceptable au terme duquel il est approprié de permettre à un autre syndicat d'exercer son droit,

2 L'affichage déjà fait, la promotion déjà accordée, l'engagement déjà effectué, etc. peuvent difficilement être repris pour se conformer à des règles nouvelles. 
s'il y a lieu, à l'accréditation ou encore, d'adopter la convention collective aux nouveaux besoins respectifs des parties. D'ailleurs, l'art. 72 al. 3 C.t. révèle bien le deuxième objectif de cette règle: éviter que les parties puissent, par connivence ou inadvertance, empêcher la venue d'un autre syndicat. Pour atteindre ce double objectif, il suffit que l'on puisse bien connaître quand la convention collective se termine. L'art. 52.2 in fine C.t. répond aussi de ce même besoin.

3. Les parties bénéficient d'une certaine liberté de manoeuvre au cours de la négociation. Plusieurs dispositions du Code du travail en témoignent d'une façon éloquente:

-. le début de la négociation est laissé à l'initiative de l'une d'elles (art. 52 C.t.). On énonce même une règle dispositive précisant que les parties peuvent entamer la négociation 60 jours après l'accréditation (art. 52 C.t.);

- les parties sont tenues de négocier, mais nullement contraintes de conclure (art. 53 C.t.); et elles sont libres de prendre le temps nécessaire pour négocier pourvu qu'elles s'entendent à ce sujet;

-. en tout temps, peuvent-elles faire intervenir un conciliateur et nullement après une durée pré-déterminée de négociation directe (art. 54 C.t.);

- bien qu'ayant acquis le droit de grève et de lock-out, elles ne sont nullement tenues de l'exercer, sous peine de déchéance (art. 58 C.t.) et peuvent s'entendre pour emprunter bien d'autres voies: poursuivre la négociation ou la suspendre, faire appel à un conciliateur ou médiateur, s'en remettre à un arbitre, etc.;

- bien que tenues de respecter un délai minimum d'un an et un maximum de trois ans (art. 65 C.t.), elles peuvent adapter la durée de la convention collective selon leur appréciation de la conjoncture compte tenu de l'importance de cet élément pour la sauvegarde de leurs intérêts communs et à la fois, respectifs;

-. le législateur confère une meilleure sécurité juridique aux parties en écartant d'avance tout danger de voir annuler leur convention collective en raison d'un vice d'une ou de plusieurs de ses composantes (art. 64 C.t.).

Malgré tous ces éléments, l'approche maintenant retenue par le tribunal du travail pourrait signifier que les parties ne peuvent plus toujours valablement convenir d'un maximum réel de trois (3) ans depuis la conclusion de leur convention collective dès qu'elles lui font porter un effet rétroactif général, formel, bien qu'il soit impossible en pratique et aussi, en logique, (point 5). Cette approche nous apparaît inappropriée, nullement nécessaire et aucunement fondée. D'abord, parce qu'elle limite la liberté de manoeuvre des parties. S'il fallut, à titre d'exemple, douze (12) mois de négociation ardue pour arriver à ce compromis et qu'il leur faut tenir compte de la situation de fait au cours de cette dernière période, pourquoi les parties seraientelles privées de se lier pour les trois (3) prochaines années si tel était leur volonté? Un syndicat concurrent ne serait pas juridiquement préjudicié de cette situation sachant tout aussi clairement quand il pourra effectivement exercer son droit à l'accréditation. Par ailleurs, les effets du retard à dépo- 
ser, par inadvertance ou collusion, sont directement et expressément prévus à ce même article 72 . Un autre syndicat peut alors valablement présenter une requête en accréditation. Bien plus, le Code du travail confère ce même droit à tout autre syndicat dès que l'on retrouve la situation visée à l'art. 22 par. c C.t.: six (6) mois après l'acquisition du droit de grève (art. 58 C.t.). Ces deux mesures législatives protégeant les droits des autres syndicats dispensaient le tribunal d'en aménager une autre, par voie interprétative.

4. L'analyse même des textes peut conduire à la conclusion qu'il faut distinguer l'acte lui-même, soit la convention collective, de ses effets. L'art. 59 C.t. n'en est-il pas une manifestation claire? Après l'expiration de l'acte, ces effets demeurent et parfois, même pour longtemps. Alors que les art. 65 et 66 C.t. traitent expressément de la durée de la convention collective, l'art. 72 porte strictement sur les effets de l'acte. Les expressions utilisées respectent cette logique juridique: il faut que l'acte soit avant de produire quelques effets. Cette rétroactivité s'effectue par voie de fiction et ne vise que les effets et non l'acte. Même lorsque les parties conviennent que tous les effets de la convention doivent rétroagir, nous savons que cette formulation elleptique ne peut signifier et comprendre que les seuls effets rétroactifs possibles $^{3}$.

5. Si l'acte juridique lui-même pouvait rétroagir, il ne s'agirait plus, à proprement parler, d'une rétro-action, mais d'une extraordinaire fiction imposant de faire comme si l'acte avait été au moment où des faits se sont produits et que les acteurs ne pouvaient alors même en connaître l'existence. Cette fiction ainsi poussée à l'extrême nous conduirait à soutenir que les effets de l'acte ne rétro-agissant pas puisque ce retour au passé est déjà le fait de l'acte entier. Les termes mêmes employés à l'art. 72 C.t. ne permettent pas une descente aussi entière dans le temps: on n'y traite que des effets de la convention collective et non pas de la convention elle-même. Par ailleurs, lorsqu'il s'agit du calcul de la durée aux art. 65 et 66 C.t., on ne traite pas de ses effets, mais bien expressément de l'acte lui-même. Retenons que le législateur a su maintenir cette distinction fondamentale lorsqu'il traite des tenants et aboutissants de la convention collective: à l'art. 72, pour le temps antérieur à l'acte, soit ses effets et à l'art. 59 al. 2 et 3 pour les effets postérieurs à l'expiration de la convention.

6. Selon l'art. 72 C.t., la convention collective serait toujours conclue sous la condition suspensive de son dépôt, mais produirait toujours un certain effet rétroactif, soit à la date de sa signature, soit à celle qui y est précisée. Un tel effet rétroactif ne se produirait pas cependant dans le seul cas où le jour du dépôt coïnciderait avec celui de la signature de la convention: situation techniquement possible mais peu probable. Lorsque les parties établissent une date à partir de laquelle les nouvelles conditions de travail auront une portée rétroactive, elles s'entendent, par cette voie rapide et facile, pour régler la situation passée. Tel est l'objet principal de l'entente et nullement celle de limiter d'autant, pour le futur, la durée de la convention collective. Â ce sujet, nous pouvons considérer deux hypothèses: ou les parties précisent la date ferme de la fin de la convention collective ou, elles

3 À deux reprises, M. le juge Lesage souligna cette limite de la rétroactivité sans poursuivre plus avant cette analyse. 
l'établissent d'une façon indirecte, en donnant le nombre d'années ou de mois de cet acte. Dans le premier cas, il suffirait, croyons-nous, que cette date de tombée soit à l'intérieur du cadre maximal de trois (3) ans depuis son dépôt, puisque, pour le temps antérieur, certains effets de la convention collective et non l'acte lui-même peuvent seul rétroagir de fait et de droit. Dans le deuxième cas, il nous paraît clair que si les parties entendent se doter d'une convention collective de 18 mois, il doit s'agir de 18 mois futurs depuis son dépôt, condition suspensive à son existence puisque c'est là la fonction principale de cette publicisation. Si ces mêmes parties ont pris douze (12) mois pour conclure cette même convention collective et qu'elle doit produire des effets rétroactifs (quelle que soit la formulation employée pt. 1) depuis le début de la négociation, il faudrait alors admettre que cette convention collective, apparemment de 18 mois, n'aurait plus que six mois de vie utile?

7. Pour inciter les parties à faire ce dépôt dans les 60 jours de la conclusion de la convention collective, on permet qu'un autre syndicat puisse être alors accrédité (art. 72 al. 3 C.t.). Notons qu'il s'agit d'une menace puisque les parties à la convention collective peuvent corriger cette lacune tant que cet autre syndicat ne s'est pas prévalu de son droit à l'accréditation. S'il fallait réduire la durée active d'une convention collective du temps de ses effets rétroactifs, nous ajouterions une sanction à celle déjà retenue à ce même art. 72, al. 3. De plus, nous irions à l'encontre d'un autre objectif recherché, soit la sécurité juridique et la stabilité des rapports des parties. Selon la formulation plus ou moins heureuse, claire et précise de la modalité traitant des effets rétroactifs, la durée de l'acte en dépendrait, la venue d'un autre syndicat serait plus ou moins certaine et le droit d'entamer la négociation de son renouvellement (art. 52 C.t.) pourrait en dépendre.

En clair, nous croyons qu'un tel compte à rebours de la durée de la convention collective n'est pas fondé en droit. Si la convention collective devait elle-même rétroagir, comment alors qualifier la grève qui l'aurait immédiatement précédée, alors que l'art. 107 prohibe un tel acte de pression au cours de sa durée? Pareille question permet, tout au moins, de constater que la fiction même juridique doit connaître des limites!

\section{La convention collective de moins d'une année est-elle nulle?}

Selon le tribunal du travail, une convention collective de moins d'une année serait nulle, d'une nullité absolue et le tribunal n'aurait pas compétence pour la prolonger jusqu'au seuil minimal d'une année complète exigé à l'art. 65 C.t. Cette rigueur peut surprendre et surtout, les motifs avancés pour la justifier: le respect de la volonté des parties, le défaut de compétence du tribunal puisqu'il n'est pas un arbitre de différend et les limites de la portée de l'art. 64 C.t. assurant la validité de la convention collective malgré «la nullité d'une ou plusieurs de ces clauses». Pourquoi ces trois motifs n'ont-ils pu nous convaincre?

Au sujet du respect de la volonté des parties, il faut reconnaître que leur liberté de manoeuvre comporte une limite précise: elle doit s'exercer dans le respect des lois et de l'ordre public (art. 62 C.t.). Or, le législateur 
établit d'autorité que la convention collective doit être «... d'au moins d'un an et d'au plus trois ans» (art. 65 C.t.). En prolongeant une convention collective, apparemment de moins d'un an, pour respecter la durée minimale légale, le tribunal du travail n'aurait fait qu'imposer d'une manière positive et utile le respect d'une disposition légale qui liait les parties et qui les empêchaient de convenir de moins. Que l'illégalité soit le fait des deux parties ou de l'une d'elles, le tribunal du travail peut et doit corriger la situation et il le fait d'ailleurs, sans aucune hésitation, en bien d'autres cas. Comment ce même tribunal du travail peut-il respecter davantage «la volonté des parties» lorsqu'il réduit, comme il l'a encore fait en cette affaire Ambulance S.O.S., en réduisant la durée excédentaire d'une convention collective pour la rapporter à son maximum de trois ans?

En quoi est-ce plus respectueux de réduire que de prolonger alors que, dans l'un ou l'autre cas, on ne fait que resituer les parties dans le cadre légal de l'art. 65 C.t.? En deuxième lieu, l'intention précise du législateur est très bien rappelée par la présomption créée à l'art. 66 C.t. à titre de mesure préventive et pour la sécurité de tous, dans le cas du silence à la convention collective au sujet de sa durée. L'expression «... au moins un an» à l'art. 65 exprime bien aussi que l'on ne peut convenir de moins. Si les parties y contredisent alors, elles dépassent la liberté conventionnelle pré-déterminée au Code du travail (art. 62) et le tribunal peut et doit la porter au seuil légal d'une année. Le commissaire du travail dispose même du pouvoir de clarifier «... la date d'expiration de la convention collective lorsque cette date n'y est pas clairement indiquée» (art. 52.2 in fine C.t.). Cette disposition illustre bien le souci de sécurité que l'on tente de fournir et aux intéressés à la négociation et à l'accréditation.

Finalement, l'art. 64 C.t. précise la non-invalidité de la convention collective «... pour la nullité d'une ou de plusieurs de ces clauses». Une telle disposition illustre, on ne peut plus, cette volonté de conférer aux parties toute la stabilité et la sécurité nécessaire en leur assurant la valeur de leur convention pour sa partie résiduelle. Ce même objectif ne sert-il pas aussi de fondement, en partie du moins, aux art. 65 et 66 C.t.? Comment alors le tribunal peut-il refuser d'appliquer l'art. 64 C.t. lorsque la convention est d'une durée moindre que le seuil minimal de l'art. 65 C.t.? Seule cette partie de l'acte serait nulle et après correction, la convention doit subsister ${ }^{4}$. L'approche proposée par le tribunal nous éloignerait beaucoup de l'économie générale du régime où on tente de conjuguer, nous l'avons déjà souligné, ce double objectif: stabilité des rapports et sécurité des parties et remplacement possible, à période fixe, du syndicat.

4 Dans ce cadre, il est vrai que le temps demeure un facteur important et toujours considéré pour l'évaluation respective du «compromis». Mais, ce temps vaut autant pour les mois amputés au terme de la troisième année que pour ceux ajoutés pour compléter l'année minimale. 